\title{
Essentials of Novel Inventory Management Systems
}

\author{
Rohitha Goonatilake $^{1} \&$ Sofia C. Maldonado ${ }^{2}$ \\ ${ }^{1}$ Department of Mathematics and Physics, Texas A\&M International University, Texas, USA \\ ${ }^{2}$ School of Engineering, Texas A\&M International University, Texas, USA \\ Correspondence: Rohitha Goonatilake, Department of Mathematics and Physics, Texas A\&M International \\ University, 5201 University Boulevard, Laredo, Texas 78041-1900, USA. E-mail: harag@tamiu.edu
}

Received: December 3, 2017

Accepted: February 10, 2018 Online Published: February 12, 2018

doi:10.5539/emr.v7n1p31

URL: http://doi.org/10.5539/emr.v7n1p31

\begin{abstract}
Keeping inventories adequately maintained to meet the demands of future sales or uses in business enterprises determines the extent of business's success. Hence, a successful inventory management is necessary, in particular, guided by the formulating of deterministic, stochastic, and probabilistic models. This essentially describes optimal inventory policies in terms of when and how to replenish the inventory. In this paper, we discuss several basic considerations used in determining inventory models that address the needs of current manufacturing and business enterprises. Theories and essentials supported by the novel approaches are integrated to reflect the representation of many actual situations. Multi-product and multi-facility models are given additional consideration at the end.
\end{abstract}

Keywords: demand, input, output, seasonal variation, inventory models, autocorrelation

\section{Introduction}

Most businesses carry materials, supplies, or products for sale or to provide input to the production process. These include stocks or items needed for production, supporting activities, and customer service in the forms of finished products and spare parts. This is constituted to be an inventory. An aggregate inventory management (AIM) is primarily concerned with managing inventories in accordance with their classifications, prices, and the purposes for which they are required. An AIM usually involves: 1) the uninterrupted flow of inventories, 2) type of inventories, 3) supply and demand trends, 4) functions they perform, 5) objectives, and 6) costs involved. Understanding how the inventory system works assists in maintaining adequate inventory for a business enterprise to survive in the turbulent economy and, at the same time, allows it to be successful. Demand of products will not be constant due to seasonal variation. Obviously, manufacturing sector needs, market volatility, and most importantly, availability of raw materials affect how this process continues. Basics of an inventory system may be described using the basic inventory model in Figure 1 for an independent demand system. In addition, raw and in-process, distribution inventories, and maintenance, repair, and operational supplies are part of inventory and flow of materials.

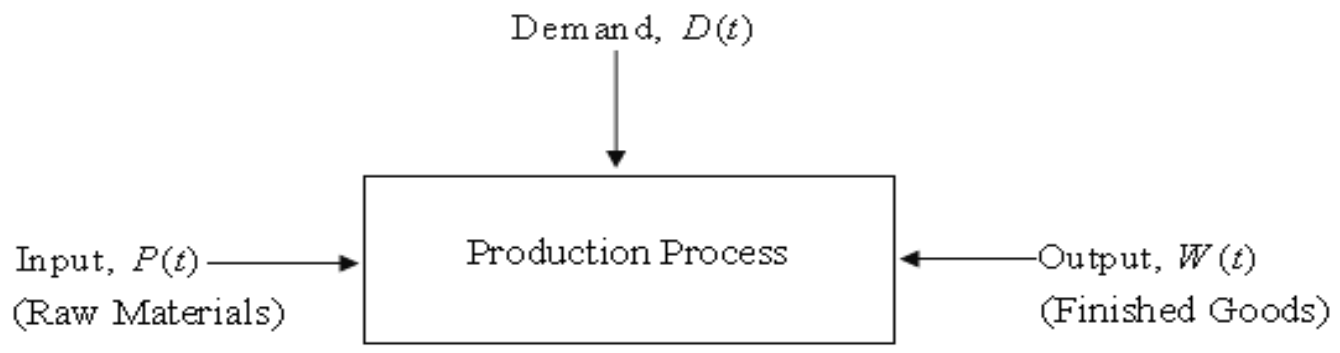

Figure 1. A basic inventory model

Total inventory at any given time is the combination of raw materials, work-in-process, and finish products. 
Total Inventory $=$ Raw Materials + Work-In-Process + Finished Products

Scientific inventory management, in particular, may include:

A). Deriving a mathematical model to suit the behavior of the inventory system.

B). Establishing an optimal inventory policy with respect to this inventory system and business forecast. Inventory policy generally consists of the types of items to be ordered together with timing decisions and quantity decisions.

C). Utilizing capabilities available from the advancement of computers to maintain a record with regards to inventory level and knowing when and how the inventories are to be replenished.

Any inventory system may be introduced naturally with profitability in mind. The determining components are: 1) the costs of ordering/manufacturing, 2) holding/storage costs, 3) penalties on unmet demand or shortages, 4) revenues from sales, 5) salvage costs, and 6) costs from discounting. Naturally, these items should be included in any reasonable models.

\section{Preliminaries}

Multi-item retail inventory system demand substitution is anticipated. Accounts of demand substitution defines everything from customer service influence on the choice of items to holding the proper amount of stock and keeping the optimal level of inventory for each item which is stocked. Although certain items are not in stock, resulting substitutions may increase the demand of other items, which affects the optimal shock levels (Smith \& Agrawal, 2000). An economic ordering quantity allows items being recovered in time. Reverse logistics is the collective effort for logistic environments with recovery of goods and materials. This has gained an increasing attention recently. The more rigid environmental legislation and growing environmental concerns is a reason for this scenario. Another reason is the economical attraction of reusing products instead of disposing of them at all (Teunter, 2001). A stochastic inventory system in a supply chain with asymmetric information is given strong consideration. Increase in stock helps reduce cycle stock by providing the supplier an additional incentive to decrease batch quantities, but it simultaneously gives the consumer an incentive to increase safety stock by expanding on backorder costs (Corbett, 2001). Information inaccuracy in inventory systems is working against stock losses and stockout. Organizations have automated their inventory management processes to rely on an information system in critical decision-making processes. However, if the information provides inaccurate data, the ability to provide larger availability of products at the minimal operating cost is definitely compromised (Kanga \& Gershwinb, 2005). Lot-size inventory is to purchase or manufacture in quantities greater than what is needed instantaneously. Lot-sizing for inventory systems with product recovery is studied. Recovered items from this process produce good-as-new items that satisfy the same demands as new items. The demand rate and return fraction are computed deterministically. Supply chain operations using both serial and distribution inventory systems are considered (Teunter, 2004). The inventory policy determines what to be ordered following a variety of business decisions, when an order is to be made that includes its timing discussions, and how much of each quantity to be ordered. There are two policy considerations based on periodic and continuous review process. The latter can involve discussions on multiple quantities on periodic and continuous basis as depicted in Figure 2 providing two possible scenarios.
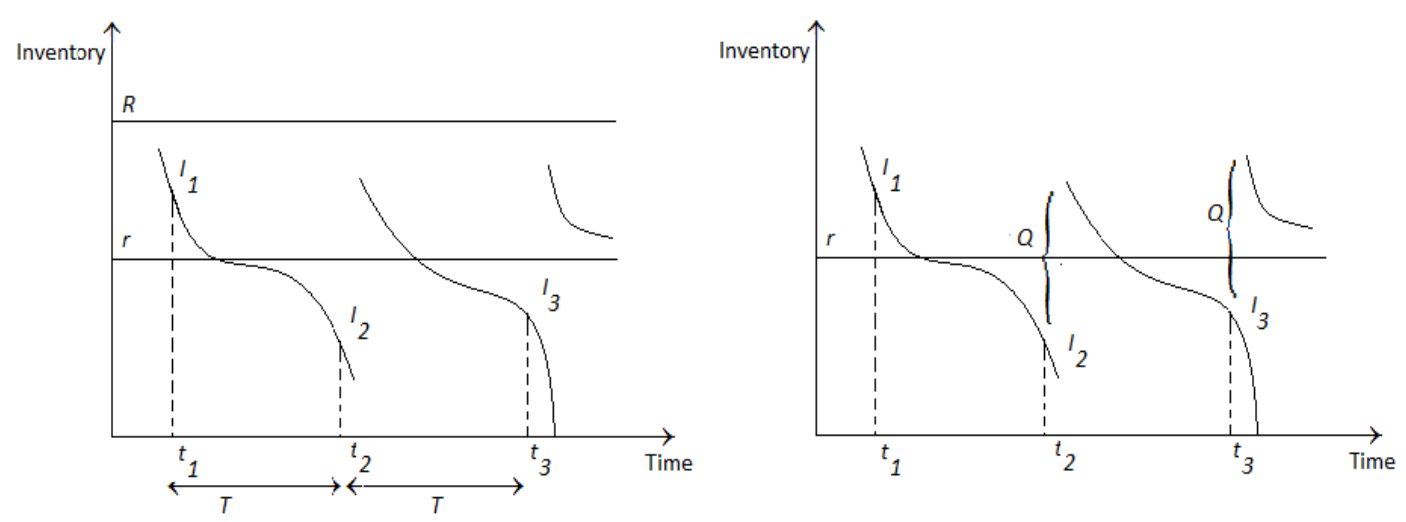

Figure 2. Graphs represent periodic (seasonal) and continuous review policies 
A common control policy consideration in connection with single-echelon inventory control is a so-called $(R, Q)$ policy, where $R$ is the reorder point and $Q$ is the batch quantity required. When the inventory position reduces to or falls below the level of $R$, an ordering is made of a number of batches of size $Q$. The resulting inventory position is strictly larger than $R$ and less or equal to the sum of $R$ and $Q$. For continuous review, continuous demand of unit will always hit the reorder point exactly when the ordering is needed, and this policy is equivalent to an $(s, S)$ policy with $s=R$ and $S=R+Q$. An optimal stock policy, order-up-to- $S$ policy, or $S$ policy means that order up to the inventory position $S$ is maintained. The discrete integral demand requires that such a policy is a special case both of an $(R, Q)$ policy with $R=S-1$ and $Q=1$, and of an $(s, S)$ policy with $s=S-1$, often denoted $(S-1, S)$ policy (Teunter, 2004).

A standard approach for policy evaluation using a new decision rule can also be formulated to consider an arbitrary time $t$ and also the time $t+L_{i}$ assuming that the inventory position (stock on hand together with outstanding orders except the backorders) at time $t$ is $k$ (Axsäter, 2003a). Accordingly, the inventory level (stock on hand-backorders) at time $t+L_{i}$ as $k-D\left(L_{i}\right)$, where $D\left(L_{i}\right)$ is the stochastic led time demand. The expected rate of holding and backorder cost at time $t+L_{t}$ is $C_{i}(k)=\left(h_{i}+b_{i}\right) e^{-\lambda_{i} L_{i}} \sum_{j=0}^{k-1}(k-j) \sum_{n=0}^{j} \frac{\left(\lambda_{i} L_{i}\right)^{n}}{n !} f_{i, j}^{n}+b_{i}\left(\lambda_{i} L_{i} \mu_{i}-k\right)$. Adding some mild assumptions to the demand distribution, we obtain the average long-run holding and backorder cost per time unit as $C_{i}=\frac{1}{Q_{i}} \sum_{k=R_{i}+1}^{R_{i}+Q_{i}} C_{i}(k)$ (Axsäter, 2003b).

In Figure 3, the applicability of the proposed approach is demonstrated. It also provides the layout of the exemplary echelon inventory system in a nutshell. This echelon inventory system comprised of six serial stages will entirely meet the customer demand (Köchel \& Nieländer, 2005).

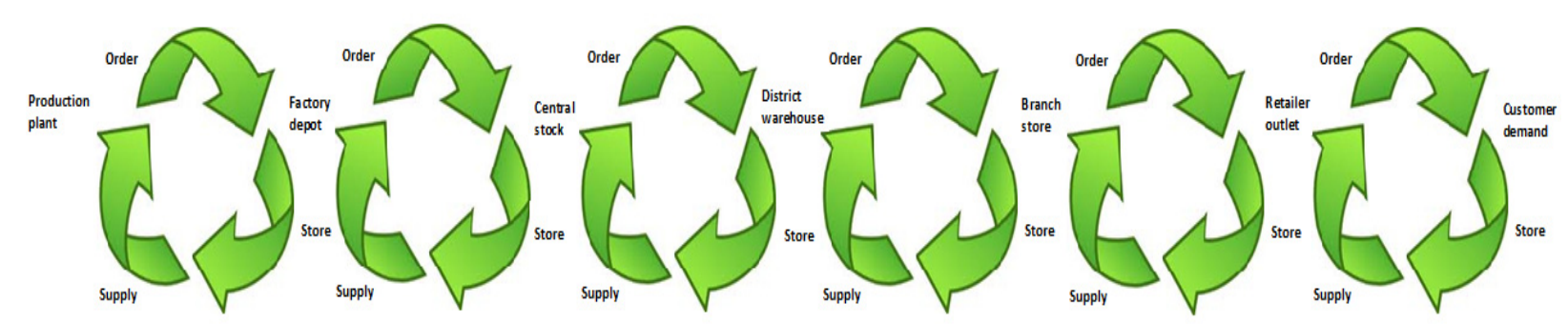

Figure 3. Layout of the exemplary echelon inventory system (Köchel \& Nieländer, 2005)

The value of advanced demand information is paramount. Advance demand information, if available and used effectively, will simultaneously improve the performance of production and management of inventory systems. 
Intuitively, better information available on future demands leads to keeping lower inventory levels for the same level of service (Karaesmen, Liberopoulos, \& Dallery, 2004). A food chain system with ratio-dependent functional response, pulse, delays, and feedback controls is considered (Jia \& Liu, 2012). The sufficient conditions of the existence and global asymptotic stability of positive periodic solution provide a good theoretical basis for the further study for the food chain system with ratio-dependent functional response and feedback controls (Daniel \& Vaaler, 2006).

\section{Deterministic Inventory Models}

First, we consider a somewhat simplified inventory model for the purpose of introducing a more general inventory model (Graves, 1999). It can be based on either deterministic or stochastic models. The deterministic models are based on actual demands resulting from economic forecast and formulating actuality of quantity required, market volatility, and movements of ongoing and future economic considerations. Let us consider a multi-period production planning under the demand of assembly lines as products are purchased from subcontractors. We consider the problem of planning the procurement of a single item when there is one opportunity to reorder in each sequence of identical-length periods and demand in any period at any given time (Graves, 1999). The effective use of e-collaboration tools in supply chain management is vital for similar actions (Ovallea \& Marquezb, 2003). The material flow is subjected to the decisions including maximizing revenue from sales, minimizing procurement, and adequacy of inventory, production, and distribution costs providing a good level of customer service, stabilizing employment levels, and holding down investment in the facilities. A simple model can represent this situation where stock levels are depleted over time. They are replenished by the arrival of new product items. The quantity, $Q$ be the item produced, which is withdrawn continuously at a constant rate $a$ in a unit time. The cycle length is $Q / a$. The production cost cycle, $C(Q)$ is $C(Q)=\left\{\begin{array}{cl}0, & \text { if } Q=0, \\ K+c Q, & \text { if } Q>0,\end{array}\right.$ where $K$ is the setup cost charged at one time of ordering, $c$ is the production cost per item, and $h$ is the inventory cost per item/time. The information of $Q, K, c$, and $h$ is changed rapidly and is needed to compute production cost. This is, in fact, a well-known economic lot-size formulation in inventory control. Almost all business enterprises are now faced with the daunting reality of managing time and optimizing production processes with the support of technology in all corners of the world. The ability of keeping track of the manufacturing progress to meet global needs in real-time can be exhaustive. A simulation approach proposed to meet the market demands has been changing rapidly. For example, cellular manufacturing techniques can possibly be employed to avoid the capital costs of keeping finished products at unnecessarily high levels (Chan \& Chan, 2007). Other issues arising from the approach need to be dealt with the property in keeping with parameters of the inventory system.

We now consider a model that is an accurate enough representation of many actual situations that include shortages and overages. Presented in Figure 4 is a basic model which does not allow shortages within the first cycle, permits shortage in the subsequent cycle, and results in an overage in the third cycle. 


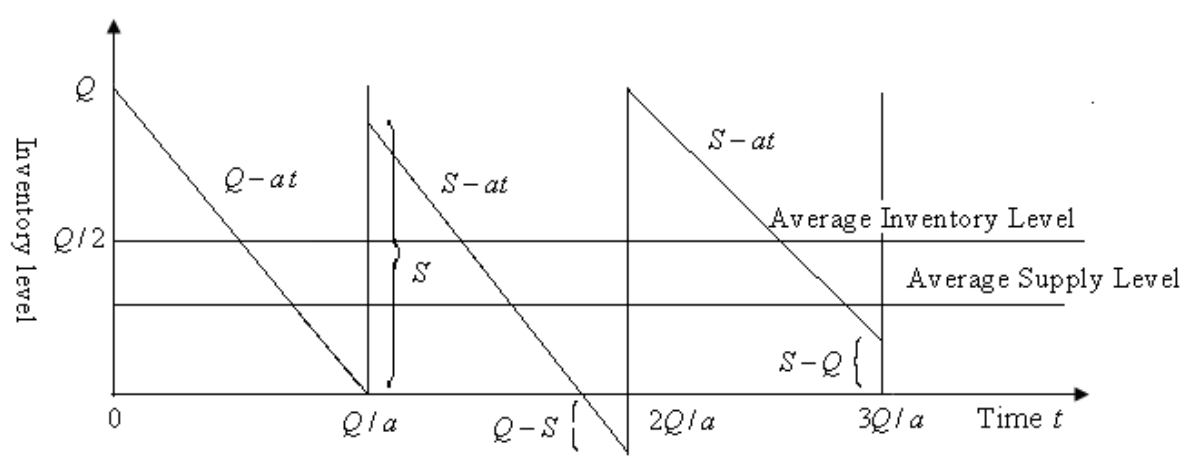

Figure 4. Inventory level with shortages and overages present

The average inventory level during a cycle is $(Q+0) / 2=Q / 2$ per unit/time resulting in a cost of $h Q / 2$ per unit time. The total holding cost per cycle is $\frac{h Q^{2}}{2 a}$, the total cost per cycle is $\frac{\left(K+c Q+\frac{h Q^{2}}{2 a}\right)}{Q / a}$, and the total cost per unit time is $\frac{a K}{Q}+a c+\frac{h Q}{2}$. If shortages are permitted, the total holding cost per cycle is $\frac{h S}{2} \cdot \frac{S}{a}=\frac{h S^{2}}{2 a}$, as computed above. If the shortages continue for a time $(Q-S) / 2$, assuming the item is priced at a cost of $p$ demand unfilled per unit, $k$ is the cost per item keeping overage items, and the total shortages cost per cycle is derived by $\frac{p(Q-S)^{2}}{2 a}$. Similarly, the total overage cost per cycle is $\frac{K(S-Q)^{2}}{2 a}$. The total cost per cycle is $K+c Q+\frac{h S^{2}}{2 a}+\frac{p(Q-S)^{2}}{2 a}+\frac{K(S-Q)^{2}}{2 a}$. The total cost per unit time is $\frac{K+c Q+h S^{2} / 2 a+p(Q-S)^{2} / 2 a+K(S-Q)^{2} / 2 a}{Q / a}$ for each cycle. By differentiating with respect to $S$ (among two variables, $S$ and $Q$ ), we obtain the shortage that will minimize the cost as $S=\frac{(p+K) Q}{(p+K-h)}$. Any other consideration that consists of a combination of multiples of shortages and overages can be formulated similarly. For example, the average annual cost, say $G(Q)$, is equal to $G(Q)=\frac{K a}{Q}+a c+\frac{h Q}{2}$ under special condition described in Figure 5. This provides a minimum value of $Q^{*}$ being equal to $Q^{*}=\sqrt{\frac{2 K a}{h}}$ that is known as the economic order quantity (EOQ). This will lead to the ratio of $\frac{G(Q)}{G^{*}(Q)}=\frac{1}{2}\left[\frac{Q^{*}}{Q}+\frac{Q}{Q^{*}}\right]$ if $c$ is small or $c=0$ (Johnson \& Montgomery, 1974 and Hillier \& Lieberman, 1967). 


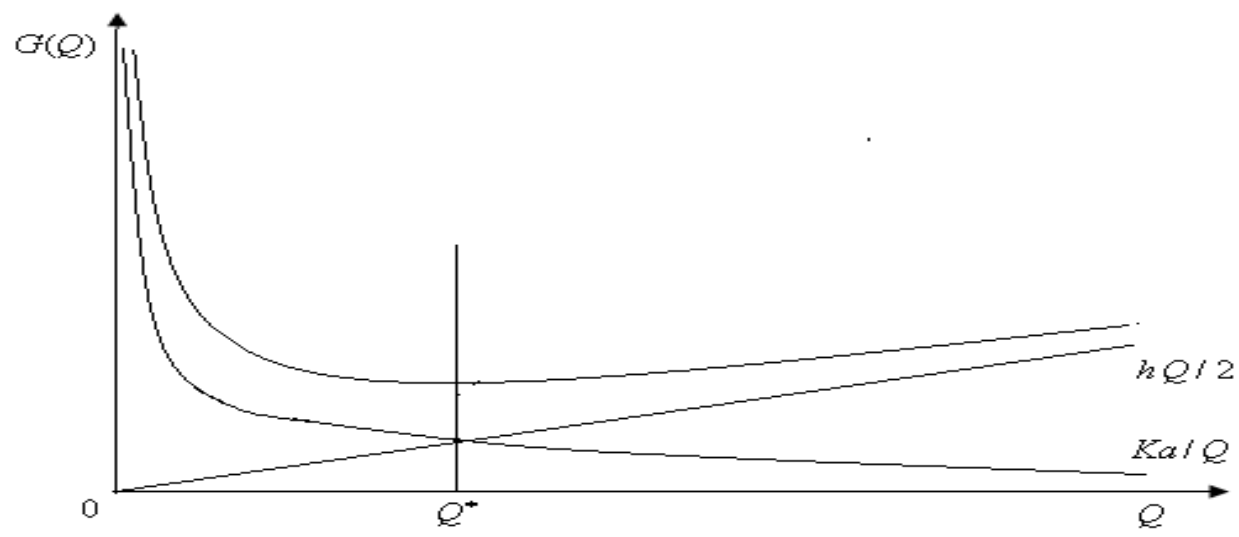

Figure 5. Graph of $\mathrm{G}(\mathrm{Q})$ vs. Q

If the reordering remains constant, we need to adopt a policy of ordering items of a fixed size whenever the inventory level diminishes. We need to determine two decision variables: the value of the items reordering and at what point this reordering should occur. A mathematical model has been developed to express the average cost function of these two decision variables, and then to calculate the reorder quantity and the point at which the order is to be made that minimizes the cost function. The maximum on-hand inventory level is $I_{\text {Max }}=Q\left(1-\frac{D}{P}\right)-b$, where $D-$ the demand rate in units per year, $P-$ the production rate in units per year, and $b$ - the maximum backorder level permitted. The average inventory over the cycle is the area under the inventory triangle over a cycle and is given by $\bar{I}=\frac{1}{T}\left\{\frac{[Q(1-D / P)-b]^{2}}{2 D(1-D / P)}\right\}=\frac{[Q(1-D / P)-b]^{2}}{2 Q(1-D / P)}$. The average backorder position over a cycle is $\bar{B}=\frac{1}{T}\left[\frac{b^{2}}{2 D(1-D / P)}\right]=\frac{b^{2}}{2 Q(1-D / P)}$. The average cost per cycle is the sum of the procurement, inventory, and shortage costs during a cycle: $A+C Q+h T \bar{I}+\hat{\pi} T \bar{I}+\pi b$. After substituting for $\bar{I}$ and $\bar{B}$, the average annual cost is $K(Q, b)=\frac{A D}{Q}+C D+\frac{i C[Q(1-D / P)-b]^{2}}{2 Q(1-D / P)}+\frac{\hat{\pi} b^{2}}{2 Q(1-D / P)}+\frac{\pi b D}{Q}$. For the optimal values of the two decision variables, $Q$ and $b$, we simultaneously solve two equations stated as $\frac{\partial K}{\partial Q}=\frac{\partial K}{\partial b}=0$. These values are, for $\begin{aligned} \hat{\pi} \neq 0, Q^{*} & =\sqrt{\frac{2 A D}{i C(1-D / P)}-\frac{(\pi D)^{2}}{i C(C+\hat{\pi})}} \sqrt{\frac{i C+\hat{\pi}}{\hat{\pi}}} \text {. If } \pi=0, \text { the minimum average annual cost is } \\ b^{*} & =\frac{\left(i C Q^{*}-\pi D\right)(1 / D / P)}{(i C+\hat{\pi})}\end{aligned}$ calculated as $K^{*} \equiv K\left(Q^{*}, b^{*}\right)=C D+\sqrt{\frac{2 A D(1-D / P) \pi}{(i C+\hat{\pi})}} \quad$ Johnson \& Montgomery, 1974 and Hillier \& Lieberman, 1967).

The value of the reorder level, $R$, was determined by $Q$. We now consider $Q$ and $R$ as independent variables. Essentially, $R$ is chosen to protect against uncertainty of demand during lead time and $Q$ is 
determined to balance out the holding cost and setup costs, if any as showcased in Figure 6 .

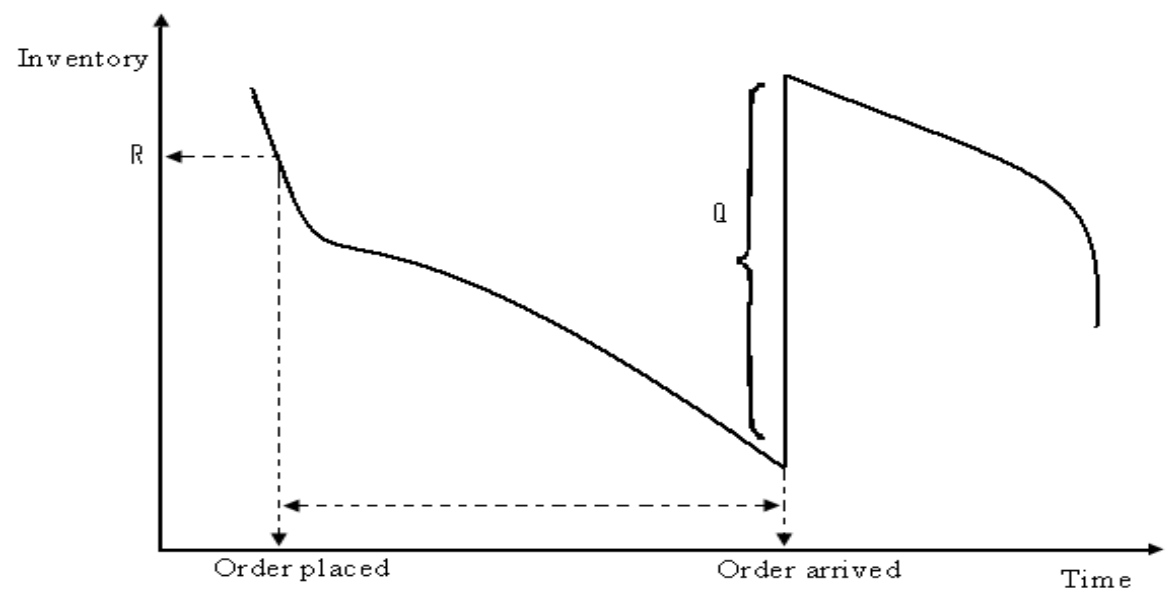

Figure 6. Inventory request over time for continuous review system

The average annual cost is now given by $G(Q, R)$ and $G(Q, R)=h\left(\frac{Q}{2}+R-\mu\right)+K \frac{\lambda}{Q}+p \lambda \frac{n(R)}{Q}$,

where $n(R)$ is the expected number of stockouts per cycle by the loss integral formula. The last term in this

model formula is due to shortage cost. The optimal values of $(Q, R)$ that minimize $G(Q, R)$ can be derived

and are equal to $Q=\sqrt{\frac{2 \lambda(K+p n(\lambda))}{h}}$ and $F(R)=1-\frac{Q h}{p \lambda}$. The optimal solution is obtained through

iterations between values of $Q$ and $R$ until the convergence occurs (Nahmias, 2009).

\section{Stochastic Inventory Models}

The inventory models in which the demand follows a probability distribution are considered next. The treatment of the inventory systems, in this case, requires the use of random variables. The multi-period models with stochastic demand that characterize the type of inventory policies $(s, S)$ seek the optimal value of $S$ and $S$. This is obtained from several complex models formulated in an attempt to suit more general situations even though a wide gap between practices and theories still exists. Suppose we want to estimate a parameter based on a finite sample of data. We can simply calculate a point or interval estimates for inventory parameters. Point estimate is to summarize the sample by a single number that is an estimate of the population parameter and an interval estimate is to find a range of values within which, we believe, the true parameter lies with a high probability (MS-0001 1922.65, 2012).

We now consider a few multi-period models. For simplicity, we consider models without setup costs together with zero lead times. The analysis to the case of positive setup costs and positive lead times can be later characterized by this simple model. Multi-period stochastic models can be formulated along these lines. The expected costs consist of three components: namely, expected cost of ordering, inventory due to holding $(H)$, and shortages, if any (assuming there is no fixed procurement cost for each item sold at $V$ ). Accordingly, the 
expected cost, $E[K \mid R]=C R+H \int_{0}^{\infty} \zeta(R, x) f(x) d x+V \int \psi(R, x) f(x) d x$, where $\zeta(R, x)$ is the excess inventory at the end of period defined by $\zeta(R, x)=\left\{\begin{array}{cc}R-x, & \text { if } x<R \\ 0, & \text { if } x \geq R\end{array}\right.$ and the shortage occurred at the end of the period is defined by $\psi(R, x)=\left\{\begin{array}{cc}0, & \text { if } x \leq R \\ x-R, & \text { if } x>R\end{array}\right.$.

The single-period stochastic mechanism can be formulated in dealing with possible shortages and overages. Let $c_{1}$ and $c_{2}$ be the loss for each unit on-hand and shortfall as a result of shortages and overages during this period, respectively. The expected loss due to possible shortages and overages is $K(R)=c_{1} \sum_{0}^{R} \int_{0}^{R}(R-D) f(D) d D+c_{2} \int_{R}^{\infty}(D-R) f(D) d D$. If $R^{*}$ minimizes $K(R)$, then $R^{*}$ satisfies the cumulative probability distribution function of demand $F\left(R^{*}\right)=\frac{c_{2}}{c_{1}+c_{2}}$ and, if we further take $c_{1}=C+H$ and $c_{2}=V-C$, this will lead to $F\left(R^{*}\right)=\frac{V-C}{V+H}$ since $\frac{d^{2} E\{K(R)\}}{d R^{2}}=(H+V) f(R)>0$. This value of $R^{*}$ leads to a minimum and $G(R)$ is a convex function; thus, the optimum policy results in $\int \operatorname{Order}\left(R^{*}-I\right)$, if $R^{*}>I$

Do not order, if $R^{*} \leq i$

For example, let us now consider a model with a fixed ordering cost. Let us assume that $A$ is charged for placing an order, and that the shortage and holding penalty costs follow a linear relationship. As such, the expected cost for stocking an amount $R \geq I$ is $\left\{\begin{array}{cl}A+C(R-I)+G(R), & \text { if } R>I \\ G(I), & \text { if } R=I\end{array}\right.$. We define $R^{*}$ as the value of the inventory level $R$ that minimizes $C R+G(R)$ and define $r^{*}$ as the smallest value of $R$ for which $C r^{*}+G\left(r^{*}\right)=A+C R+G\left(R^{*}\right)$. It is evident from Figure 7 that the cost is minimized if we order $\left(R^{*}-I\right)$ for a model with a fixed ordering cost. 


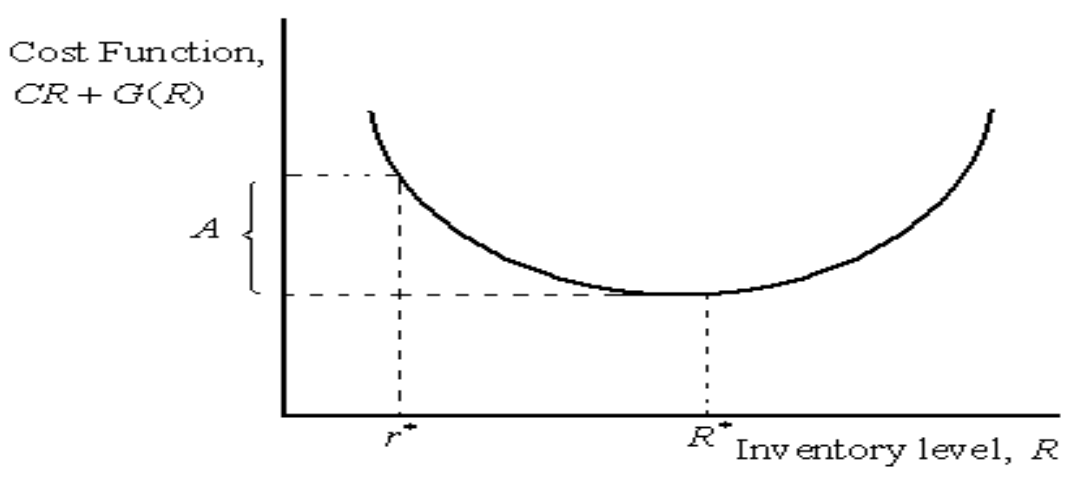

Figure 7. Graph of cost function, $C R+G(R)$ vs. $R$

\section{Inventory Forecasting}

There can be two types of estimates for inventory forecasting. One is the point estimate, in which a possible single value for the estimate, and the other is an interval estimate providing an average possible range of values. First, we present five point estimates for the inventory forecasting.

It is essential for forecasting future inventory requirements of products primarily from past data available based on statistical forecasting techniques to assess the sustainability of production process. The fact that the sales occurred during a sequence of time periods make them to be random variables. Let $X_{1}, X_{2}, \cdots$ be a sequence of random variables having the expected values, $E\left(X_{1}\right), E\left(X_{2}\right), \cdots$ which may or may not be independently identically distributed according to a given probability distribution. Based on this information, there are several potential forecasting procedures that can be used in the case of inventories.

1. Last values: Let $\hat{E}\left(X_{t}\right)$ be the forecast for subsequent periods. For this estimate, it may be used the last value, $x_{t}$, to be the estimate in this method so that this estimator is $\hat{E}\left(X_{t}\right)=x_{t}$. This estimating technique is disadvantageous causing a large variance as a result of selecting a sample of size 1 .

2. Average: This estimate may be chosen to be $\hat{E}\left(X_{t}\right)=\sum_{t=1}^{t} \frac{x_{i}}{t}$. This can be better estimator if the process is entirely stable. The use of a large amount of data can cause occasional shifts, when a reasonable estimate is desired.

3. Moving average: This estimate only uses the relevant recent data for the last $n$ periods, but is updated periodically placing much weight on $x_{t-n+1}$ compared to $x_{t}$. Accordingly, the estimate is $\hat{E}\left(X_{t}\right)=\sum_{i=t-n+1}^{t} \frac{x_{i}}{n}$.

4. Exponential smoothing: This estimate is, in fact, the weighted sum of the last observation and the one before using the smoothing constant, $\quad \alpha, \quad 0<\alpha<1$. As such, the recursive relationship $\quad \hat{E}\left(X_{t}\right)=\alpha x_{t}+(1-\alpha) \hat{E}\left(X_{t-1}\right) \quad$ can $\quad$ be $\quad$ expressed alternatively as $\hat{E}\left(X_{t}\right)=\alpha x_{t}+\alpha(1-\alpha) x_{t-1}+\alpha(1-\alpha)^{2} x_{t-2}+\cdots$.

$\operatorname{Var}\left(\hat{E}\left(X_{t}\right)=\alpha^{2} \sigma^{2}+\alpha^{2}(1-\alpha)^{2} \sigma^{2}+\alpha^{2}(1-\alpha)^{4} \sigma^{2}+\cdots=\alpha^{2} \sigma^{2} \sum_{n=0}^{\infty}(1-\alpha)^{2 n}=\sigma^{2} /(2-\alpha / \alpha)\right.$. The choice 
of $\alpha$ is done so that the variance is statistically equivalent to a moving average with as many as $(2-\alpha) / \alpha$ of observations (Hillier \& Lieberman, 1967). One main drawback of this estimate is that the process can be several periods behind in the wake of increasing mean.

5. Exponential smoothing adjusted for trend: Let us assume that $E\left(X_{t}\right)$ is linear with the slope $S$ (a trend factor). The expected rate of sales rate can either be increasing or decreasing. The estimate is $\hat{E}\left(X_{t}\right)=\alpha x_{t}+(1-\alpha)\left[\hat{E}\left(X_{t-1}\right)+S\right\rfloor$ Since $S$ is not generally known, it is estimated once again using exponentially smoothing to obtain $\hat{E}\left(S_{t}\right)=\beta\left[\hat{E}\left(X_{t}\right)-\hat{E}\left(X_{t-1}\right)\right]+(1-\beta) \hat{E}\left(S_{t-1}\right)$, where $0<\beta<1$ and $\beta \neq \alpha$ is becoming another smoothing constant. Accordingly, the new estimate of $E\left(X_{t}\right)$ is $\left.\hat{E}\left(X_{t}\right)=\alpha x_{t}+(1-\alpha) \mid \hat{E}\left(X_{t-1}\right)+\hat{E}\left(S_{t-1}\right)\right\}$

Let us assume that the inventory sequence is $x_{1}, x_{2}, \ldots, x_{n}$ with associated probabilities $p_{1}, p_{2}, \ldots, p_{n}$. The mean and variance of inventory would be $E[X]=\sum_{i=1}^{n} x_{i} \cdot p_{i}$ and $\operatorname{Var}[\mathrm{X}]=\sum_{i=1}^{n}\left(x_{i}-E[X]\right)^{2} \cdot p_{i}$. As for the confidence interval estimates, the simulations of stochastic inventory systems with finite number of historical demand data should capture the demand parameter uncertainty not only for limited data sets with size $n \leq 50$ observations, but also for data sets with size $n \geq 100$ observations for the confidence intervals of the mean service levels to attain high level of coverage. The values of $\bar{x} \pm z_{\alpha / 2}=\frac{\sigma}{\sqrt{n}}$ provide a $(1-\alpha) \%$ confidence interval estimate of the mean service levels of inventory systems. The use of $t$-distribution follows similarly (Bethea \& Rhinehart, 1991). However, it has been also considered the use of the discrete-event stochastic simulation for estimating the mean service level and the confidence interval of an inventory system in the absence of demand data. Availability of limited historical demand data and obtaining the mean service level and its confidence interval around the demand parameters estimated from historical demand data of finite length was assumed for these calculations (Johnson \& Montgomery, 1974).

Additional variables are needed to describe a complete system such as $I(t)$ - the on hand inventory level, $B(t)$ - the back order level, $O(t)$ - the on order position, $X(t)$ - the net inventory, $Y(t)-$ the inventory position at time $t$. The inventory position of the system $X(t)$ can be expressed as $X(t)=I(t)-B(t)+O(t)$. If the inventory is out of stock $(I(t)=0)$, any demand is considered a shortage. Assuming all shortages are all back ordered together with disposition of shortages, we have $Y(t)=Y(0)+\int_{0}^{t}[P(u)-D(u)] d u$, where $Y(0)$ is the net inventory level at time $t=0$. We also have $I(t), B(t)$, and $W(t)$ defined as $\begin{aligned} I(t) & =\max [0, Y(t)] \\ B(t) & =\max [0,-Y(t)]\end{aligned}$, and $P(t)$, the rate at which materials is added to the 
system and withdrawn from the system at time, $t=0$. Accordingly, $W(t)=\left\{\begin{array}{cll}D(t) & \text { if } & \mathrm{Y}(\mathrm{t})>0 \\ 0 & \text { if } & \mathrm{Y}(\mathrm{t})=0 . \\ \mathrm{P}(\mathrm{t}) & \text { if } & \mathrm{Y}(\mathrm{t})<0\end{array}\right.$ If all shortages are lost to the system, then $B(t)=0$ and $Y(t)=I(t)=I(0)+\int_{0}^{t}[P(u)-W(u)] d u$.

\section{Probabilistic Inventory Models}

Inventories undergo seasonal variation, thus enabling us to use time series analysis in inventory management. There are two types of seasonal variations. One is that the magnitude of the seasonal variation does not change on time variable. The other is the magnitude of the swing exhibits either increasing or deceasing seasonal variation. The later can be transformed to display a constant seasonal variation using the common transformation of the forms such as $y^{*}{ }_{t}=y^{\lambda}{ }_{t}$, where $0<\lambda<1$ or $y^{*}{ }_{t}=\ln y_{t}$. The importance of forecasting is necessary for businesses to grow in the turbulent economy that exhibits a great deal of market fluctuation (Bowerman \& O'Connell, 1993). The various statistical techniques that can be used to produce forecasts clearly demonstrate the necessity of using forecasts to make intelligent decisions in marketing, finance, personnel management, production scheduling, process control, and strategic management. The models for most products generally follow a sectional variation. The model $z_{t}=\delta+a_{t}-\theta_{1, L} a_{t-L}-\theta_{2, L} a_{t-2 L}-\theta_{Q, L} a_{t-Q L}$ is called the

seasonal moving average model of order $Q$. This nonzero autocorrelations is at lags $L, 2 L, \ldots, Q L$ and zero autocorrelations everywhere. If, for the time series values The first-order autoregressive process is defined by $\varepsilon_{t}=\phi_{1} \varepsilon_{t-1}+a_{t}$ in usual notations.

Dynamic programming is a very useful technique for making a sequence of interrelated decisions similar to the working of control mechanisms. Formulating an appropriate recursive relationship for this control system is an overwhelming task. Dynamic programming assures great computational savings over an exhaustive numeration in finding the best possible decisions, especially for large organizations that involve many components such as multi-period inventor processes. In addition, storage is meant to hold the inventory until it is sold or used. The holding or storage costs represent the costs associated with storage. They include the cost of capital tied up, space, insurance, protection, and taxes related to storage. Optimal results require keeping tabs on these items periodically. The asset pricing model is one example if the business heavily relies on market performances. This model will explain the variation on yield rates on various types of investments such as common stocks and corporate-bonds as usually seen in most of investments (Daniel \& Vaaler, 2006). This contributes to the losses due to inventory. The discounting risky liabilities are often prevalent in determining the expected value of price of stock. The inventory models require that demand be replenished during any period of time and be known with an amount of uncertainty. On the other hand, for the single-period inventory models, a problem is ended upon a single ordering decision being made prior to subsequent periods for uncertain demand that incorporates the important concepts of safety stock and service levels; an example of this is the periodic review (R, S) model, etc. Supply chain inventory from raw materials to final products that are available in the retail stores has many layers in between. Inventories at each stage need to be determined for the entire process to be working. Inventory cost can be a huge burden for profitability of an organization that include carrying costs, setup costs, ordering costs, and shortages simply because of lapses in the supply chain management (Ovallea \& Marquezb, 2003). Shortage cost is something that needs attention. If we cannot keep up with the demand of the customers, they will not 
come back as we will lose them. Let us assume that there is a constant loss, $\pi$, associated with each item requested as a result of the inventory being out of stock. If $K_{t}$ is the economic loss in period $t$ resulting from a given inventory not maintained adequately and $j$ is the rate of interest per each period, the discounted cost of such a policy over the period $T$ is $\sum_{t=1}^{T} K_{t}(1+j)^{-t}$ (Kellison, 1991). The discounted value by the annuity or capital recovery (factor) $\left[\sum_{t=1}^{T} K_{t}(1+j)^{-t}\right]\left[\frac{j(1+j)^{T}}{(1+j)^{T}-1}\right]=\hat{K}$, where $\hat{K}$ is the uniform cost per period. Also, note that $\sum_{t=1}^{T}(1+j)^{-1}=\frac{(1+j)^{T}-1}{j(1+j)^{T}}$. This is derived from the continuous analogue of sum of power series. That is, if the inventory holding cost is assumed to be proportional to average inventory, $I=\frac{1}{T} \int_{0}^{T} I(t) d t$.

\section{Raw Materials to Final Products}

We now briefly look into a process in which a single item is produced by a sequence of production steps needed for processing raw materials into, first, several semi-finished materials and then into a finished product to be used by the customers. An assumption is made to the fact that the raw materials inventory never ran out. For nonphysical items of this type, most of the other main cost areas with traditional physical stock are also absent from inventories, such as storage and handling costs. Similar inventory is never used up nor out of stock in general. It can drop value, however, when it reaches obsolete, or competitive the market prices naturally vary and are subject to market turbulences. This process can be mostly centered on the concept of packaging that causes adding the value to the product, the key structure behind the development of the product involving labor. Another manufacturing process that involves labor is significance for construction and gathering of the resource needed to make a final product. Ultimately, it is foreseen that the demand for specialized information products of credible information and market demand and domestic trade policies are the key to what managers of today will seek out for this manufacturing process to be a worthwhile effort (Abrigo, 2009). There can be a situation in which raw materials are part of inventory.

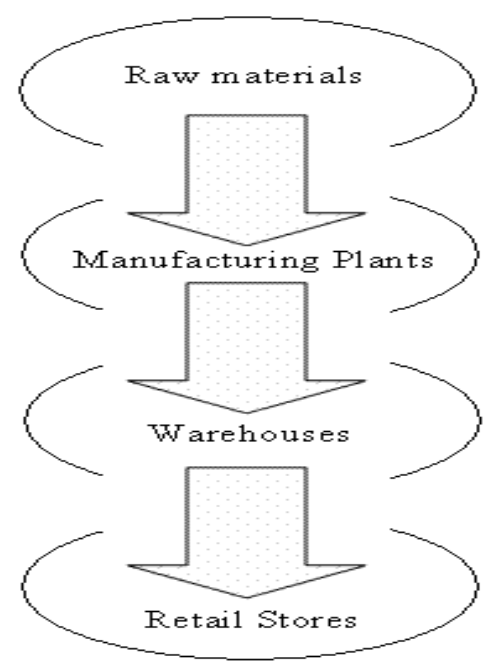

Figure 8. Single item production process

The model described emanates from the inventory process of commands whose demand occurs during a relatively short interval, after which the data becomes obsolete or is not in demand for a long period (Johnson \& Montgomery, 1974). The unique characteristic of this model is that there is only one opportunity for collecting the precise set of data at the start of any user activities. 


\section{Multi-product and Multi-facility Models}

Finally, the problem of facilitating demand arising from multiple products to multiple production locations with finite capacity and load-dependent lead times can be a daunting task. For all intended purposes, multi-stage and multi-facility models are prevalent and abundant in the industry. The characteristics of multi-stage and multi-facility models are provided to describe an actual situation. Items are produced at several factories and distributed to many facilities. A single finished product is manufactured in three stages. The problem is to choose $\left\{X_{i t}\right\}$ and $\left\{Y_{i t}\right\}$ to provide the total cost of production and inventory during the planning $Z=\sum_{t} \sum_{i}\left\{C_{i t} X_{i t}+C_{i t}^{\prime} Y_{i t}+h_{i t} I_{i t}\right\}$ subject to the appropriate conditions on nonnegative quantities, $X_{i t}, Y_{i t}, I_{i t}$ in the production process.

Let us first assume that each item is produced by several subsequent processes in each period. Let $X_{i, j, t}$ be the number of units of product $i$ produced by routine $j, j=1,2, \cdots, J_{i}$ in period $t$, and $a_{i, j, k}$ be the number of units of resources $k$ required to produce one unit of product $i$ by the process $j$. The production cost and the inventory cost, respectively, are $H_{j, t}\left(I_{j, t}\right)$ and $C_{j, t}\left(X_{j, t}\right)$. Let $X_{j t}$ be the production at serial multi-facility system facility $j$ in period $t$ and $I_{j t}$ be the inventory facility $j$ at the end of period t. The total cost during $Z=\sum_{t=1}^{T} \sum_{i=1}^{n}\left[\sum_{j=1}^{J_{i}} C_{i, j, t} X_{i, j, t}+k_{i, t} I_{i, t}^{+}+\pi_{i, t} I_{i, t}^{-}\right]$.

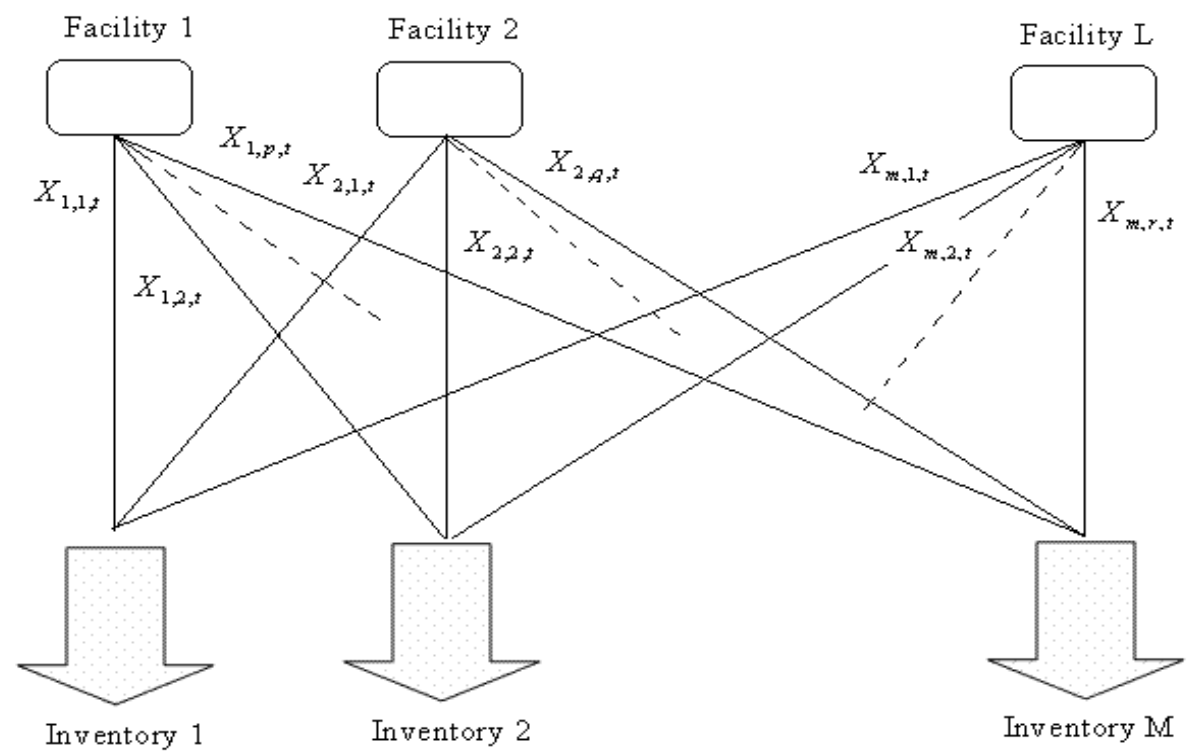

Figure 9. Multi-product and multi-facility models

The methods of linear programming are used to identify optimal maximum or minimum values of the function. It is generally used in making business decisions for practical situations that involve, decision-making analysis, 
and many other problems that can be done using a computer. Constraints are supplemented depending on availability of different resources written as linear inequalities. The constraints can be in the form of resources like the number of workers, production capacities, availability of number of machines, raw materials, etc. By using the corner point theorem, optimal solution(s) for the problem can be derived (Köchel \& Nieländer, 2002). The corner point theorem says that if a maximum or minimum value to exist, it will occur at a corner point of the corresponding feasible region.

At the end, a typical manufacturer looks into keeping the profit margin maintained and customers' needs are catered to the fullest satisfaction. The model displayed in Figure 9 adequately describes the underlined components generally found in multi-product and multi-facility models for further exploration.

\section{Conclusions}

Several simplified models, somewhat mathematically enriched models, are presented in this paper for the purpose of serving the needs of the public and manufacturers, but they all serve as the initial step of introducing rather simplified models to be part of techniques to a larger complicated inventory model.

These are sufficiently accurate presentations of many actual situations frequently used in practice. Availability of continued growth of technology and modernized computerization of inventory data (and records) processing together with rapid growth in scientific inventory management systems will benefit all concerned, the general public, and more importantly, the manufacturers and retailers.

\section{Acknowledgments}

The preliminary work of this project has been presented at the 2013 Western Hemispheric Trade Conference held on April 10-12, 2013 at Texas A\&M International University (TAMIU), Laredo, Texas.

\section{References}

Abrigo, Christine Manglal-lan. (2009). From Raw Materials to End Product: Developing an Online Information Resource for The International Labor Organization. Journal of Philippine Librarianship, 1, 13.

Axsäter, S. (2003a). Supply Chain Operations: Serial and Distribution Inventory Systems, Handbooks in Operations Research and Management Science, 11, 525-559. https://doi.org/10.1016/S0927-0507(03)11010-9

Axsäter, S. (2003b). A New Decision Rule for Lateral Transshipments in Inventory Systems. Management Science, 49(9), 1168-1179. https://doi.org/10.1287/mnsc.49.9.1168.16568

Bethea, R. M., \& Rhinehart, R. R. (1991). Applied Engineering Statistics (Statistics: A Series of Textbooks and Monographs). CRC Press.

Bowerman, B. L., \& O'Connell, R. T. (1993). Forecasting and Time Series: An Applied Approach (3rd ed.), Duxbury Thomson Learning.

Chan, F. T. S., \& Chan, H. K. (2007). A simulation approach for managing manufacturing processes and inbound logistics: a case study. Int. J. Business and Systems Research, 1(1), 115-134. https://doi.org/10.1504/IJBSR.2007.014778

Corbett, C. J. (2001). Stochastic Inventory Systems in a Supply Chain with Asymmetric Information: Cycle Stocks, Safety Stocks, and Consignment Stock. Operations Research, 49(4), 487-500. https://doi.org/10.1287/opre.49.4.487.11223

Daniel, J. W., \& Vaaler, L. J. F. (2006). Mathematical Interest Theory (5th ed.). Prentice Hall.

Graves, S. C. (1999). A single-item inventory model for a non stationary demand process. Journal of Manufacturing \& Service Operations Management, 1, 50-61. https://doi.org/10.1287/msom.1.1.50

Hillier, F. S., \& Lieberman, G. J. (1967). Operations Research (2nd ed.), Holden-Day, Inc., San Francisco, CA.

How Much Data Are Needed: Estimation of Inventory Service Levels with Limited Historical Demand Data, Unpublished manuscript MS-0001 1922.65 submitted to Management Science.

Jia, J.-W., \& Liu, J. F. (2012). A Food Chain Model with Ratio-dependent Functional Response, Impulsive Perturbations and Feedback Controls. British Journal of Mathematics \& Computer Science, 2(3), 126-136. https://doi.org/10.9734/BJMCS/2012/1230

Johnson, L. A., \& Montgomery, D. C. (1974). Operations Research in Production Planning, Scheduling, and Inventory Control. John Wiley \& Sons, Inc. 
Kanga, Y., \& Gershwinb, S. B. (2005). Information Inaccuracy in Inventory Systems: Stock Loss and Stockout. IIE Transactions, 37(9), 843-859. https://doi.org/10.1080/07408170590969861

Karaesmen, F., Liberopoulos, G., \& Dallery, Y. (2004). The Value of Advance Demand Information in Production/Inventory Systems. Annals of Operations Research, 126(1-4), 135-157. https://doi.org/10.1023/B:ANOR.0000012278.41301.24

Kellison, S. G. (1991). The Theory of Interest by Second Edition, Irwin/McGraw-Hill, a division of the McGraw-Hill Companies.

Köchel, P., \& Nieländer, U. (2002). Defining Optimal Policies in Multi-Echelon Inventory Systems: The Simulation Optimization Approach. Pre-Prints of the Twelfth International Working Seminar on Production Economics. Kongresszentrum Igls, Innsbruck, 1, 165-173.

Köchel, P., \& Nieländer, U. (2005). Simulation-based optimization of multi-echelon inventory systems, International Journal of Production Economics. Proceedings of the Twelfth International Symposium on Inventories, 93-94, 505-513

Nahmias, S. (2009). Production and Operations Analysis (6th ed.), McGraw-Hill Education International Edition

Ovallea, O. R., \& Marquezb, A. C. (2003). The effectiveness of using e-collaboration tools in the supply chain: an assessment study with system dynamics. Journal of Purchasing \& Supply Management, 9, 151-163. https://doi.org/10.1016/S1478-4092(03)00005-0

Smith, S. A., \& Agrawal, N. (2000). Management of Multi-Item Retail Inventory Systems Demand Substitution. Operations Research, 48(1), 50-64. https://doi.org/10.1287/opre.48.1.50.12443

Teunter, R. H. (2001). Economic Ordering Quantities for Recoverable Item Inventory Systems, Naval Research Logistics (NRL). John Wiley \& Sons, Inc., 48(6), 484-495.

Teunter, R. H. (2004). Lot-sizing for inventory systems with product recovery. Computers \& Industrial Engineering, 46(3), 431-441. https://doi.org/10.1016/j.cie.2004.01.006

\section{Copyrights}

Copyright for this article is retained by the author(s), with first publication rights granted to the journal.

This is an open-access article distributed under the terms and conditions of the Creative Commons Attribution license (http://creativecommons.org/licenses/by/4.0/). 